Résumés des conférences et travaux

\title{
Typologie linguistique
}

Isabelle Bril

\section{OpenEdition \\ Journals}

Édition électronique

URL : https://journals.openedition.org/ashp/2757

DOI : $10.4000 /$ ashp. 2757

ISSN : 1969-6310

Éditeur

Publications de l'École Pratique des Hautes Études

\section{Édition imprimée}

Date de publication : 1 septembre 2018

Pagination : 415-418

ISSN : 0766-0677

\section{Référence électronique}

Isabelle Bril, «Typologie linguistique », Annuaire de l'École pratique des hautes études (EPHE), Section des sciences historiques et philologiques [En ligne], 149 | 2018, mis en ligne le 11 juillet 2018, consulté le 03 août 2021. URL : http://journals.openedition.org/ashp/2757 ; DOI : https://doi.org/10.4000/ashp.2757 


\title{
TYPOLOGIE LINGUSTIQUE
}

\author{
Directeur d'études : $\mathrm{M}^{\text {me }}$ Isabelle BRIL
}

Programme de l'année 2016-2017 : Typologie linguistique. Typologie et évolution des langues austronésiennes.

La première heure de conférence développe des thématiques de typologie linguistique générale et met en perspective invariants et variation linguistique. L'analyse adopte une approche comparative, synchronique et diachronique, et aborde des faits choisis dans diverses familles des langues.

La seconde heure de conférence porte plus spécifiquement sur des systèmes et faits linguistiques observés parmi les langues austronésiennes.

\section{Première heure de conférence}

Les concepts fondateurs de la typologie sont présentés à partir des travaux de Greenberg (1960, 1966), Comrie (1981), Hagège (1982), Hawkins (1983, 2004), Croft (1990).

Cette année, l'accent est mis sur la morphologie (flexionnelle et dérivationnelle). Les limites de la typologie traditionnelle, qui est plus descriptive que prédictive, sont présentées.

A typology is not of much value unless it predicts other things about the various types of languages. [...] A typology in terms of isolating, agglutinating and fusional does not seem to correlate with anything else in the morphology at all. [...] The value of such typology is thus doubtful. (Bauer $1988: 170$ )

Une alternative à cette typologie est présentée à partir des travaux de Bickel \& Nichols, Inflectional morphology, dans Shopen 2007; Bickel \& Nichols dans Dryer \& Haspelmath (éd.) 2011, WALS; Baerman et al., Morphological complexity, 2017.

Certaines langues présentent souvent des types mêlés, comme les langues sémitiques, qui allient morphologie gabaritique, introflexive et affixale. Le cas du turc est abordé plus en détail, sa morphologie concaténative est soumise à l'harmonie vocalique, en fonction des traits \pm arrière, \pm arrondi, \pm ouvert des voyelles de la racine. L'harmonie vocalique s'exprime sur tous les morphèmes composant un énoncé, comme dans les cas suivants :

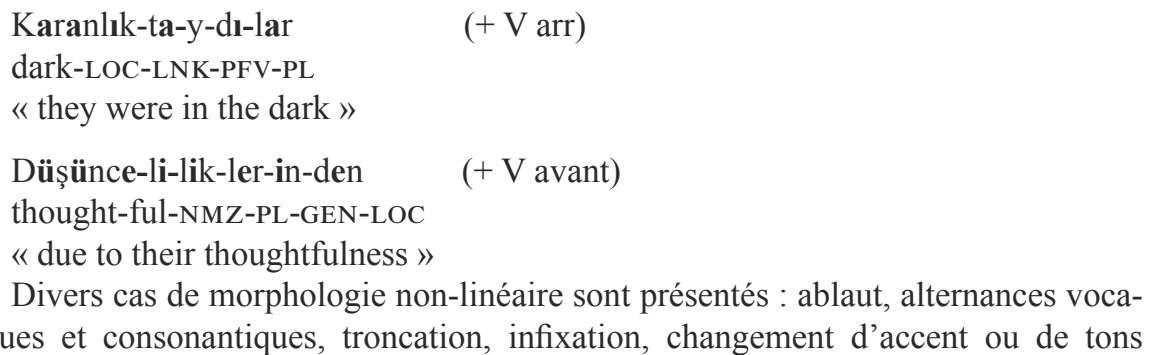
liques et consonantiques, troncation, infixation, changement d'accent ou de tons 
(langues mandingues, Creissels), ainsi que des cas de morphologie introflexive comme ceux des langues sémitiques, et des exemples de morphologie gabaritique comme en cupeño (uto-aztèque, McCarthy 1984).

La morphologie stratifiée est illustrée par des exemples empruntés à l'amis (formosan, austronésien, Bril);
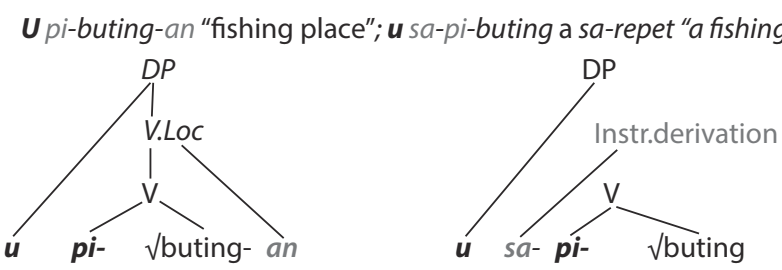

L'universel 28 de Greenberg (1963) est mis en perspective. Selon cet universel, « If both the derivation and inflection follow the root, or they both precede the root, the derivation is always between the root and the inflection ».

Est également présenté l'invariant dégagé par Greenberg selon lequel : « If a language has separate morphemes for number and case, and if both are either prefixes or suffixes, the number morpheme almost always occurs closer to the base than the case morpheme. » Les raisons d'un tel état de choses sont discutées (Bybee 1985, Corbett 2000).

L'on s'est également intéressé aux phénomènes de copies de morphèmes, tels que ceux décrits dans certaines langues australiennes, et qui indiquent des frontières de constituants comme en Kayardild (Dench \& Evans 1988).
maku
yalawu-jarrra yakuri-naa
woman catch-PAST fish-ABL(PRIOR)
« The woman caught the fish
[[[dangka-karra]-nguni]-naa $]$ mijil-nguni-naa.
[[[man-GEN-] INSTR]-ABL(PRIOR)] net-INSTR-ABL(PRIOR)
with the man's net »

\section{Deuxième heure de conférence}

Le deuxième volet de la conférence porte plus spécifiquement sur la vaste famille austronésienne. Cette famille comporte de nombreux sous-groupes: formosan, malayo-polynésien (incluant par exemple les langues des Philippines, le malgache, l'indonésien), les langues micronésiennes, les langues océaniennes de Mélanésie (Salomons, Vanuatu, Nouvelle-Calédonie, etc.) et de Polynésie.

Nous nous sommes intéressés à la phylogénie des langues austronésiennes et aux méthodes de reconstruction et de classification, telles que les correspondances phonétiques régulières, l'identification des innovations partagées (phonétiques, lexicales, morphologiques) permettant d'identifier les sous-groupes. Les limites de ces méthodes dans les situations de chaînes dialectales ont également été évoquées. Des étymons proto-austronésiens, proto-malayo-polynésiens et proto-océaniens ont été 
comparés et discutés, afin de distinguer et d'illustrer les cas d'héritage, d'innovations et d'emprunts.

Cette famille est un vaste laboratoire d'étude pour ce qui a trait à l'évolution linguistique, la diversité typologique et la variation.

\section{Typologie des constructions possessives dans les langues océaniennes}

Une typologie des constructions possessives dans les langues océaniennes a été présentée par V. Alfarano. Quelques grands traits ont ainsi été discutés, tels que la distinction entre possession aliénable et inaliénable, ou entre possession dominante (marquée par $a$ ) ou subordonnée (marquée par $o$ ). Certaines langues océaniennes font usage de classificateurs possessifs, dont certains sont reconstruits en proto-océanien : *ka- (nourriture), *m(w)a- (boisson), *na- (général) [Pawley 1973, Lichtenberk 1985].

\section{Le système des voix de l'amis et des langues formosanes}

Les langues austronésiennes occidentales sont connues pour leur système de voix multiples. L'amis, comme d'autres langues formosanes, et comme les langues des Philippines, possède des voix distinctes orientées vers l'acteur, le patient, le lieu, l'instrument, le bénéficiaire. Leur système d'alignement est parfois scindé (ergatif ou accusatif), c'est le cas de l'amis. Ce système se manifeste aussi bien dans les énoncés realis qu'irrealis où les morphèmes prennent des formes différentes.

Ce système de voix se simplifie et devient résiduel dans l'aire océanienne.

Hiérarchie de l'accession des expressions indéfinies aux fonctions actancielles et types d'encodage de l'indéfini

Nous avons également abordé la hiérarchie d'accès aux fonctions actancielles des expressions indéfinies dans diverses langues austronésiennes, traitant ainsi de l'incorporation de l'objet générique ou indéfini, qui ne compte plus comme un argument, et qui équivaut à une intransitivisation (en nêlêmwa de Nouvelle-Calédonie, par exemple).

En amis, un terme indéfini non-spécifique ne peut accéder à la fonction de sujet ou de pivot syntaxique; pour éviter cet état de fait, des constructions existentielles ou des nominalisations sont requises, telles que la suivante.

$\mathrm{Si}$ mi-kungkung-ay t-u panan.
there.is AV-knock-NMZ
«Somebody is knocking at the door. » (lit. there's a knocker at the door.) [Bril]

En amis comme en tagalog, des changements de voix prennent le terme défini comme pivot, et évitent ou rétrogradent le terme indéfini. En tagalog (ex. suivants), la voix patient (b) est requise lorsque le patient est défini, alors que la voix acteur (a) est choisie si le patient est indéfini et l'acteur défini. Enfin, si aucun argument n'est défini, ni au minimum spécifique, une construction existentielle est requise.
a. $\mathrm{B}<$ um $>$ ili ng bangka ang mangingisda.
buy $<\mathrm{AV}>$ GEN boat NOM fisherman
«The fisherman bought a boat. » specific indef. (Schachter \& Otanes, 1972 : 70)
b. $\mathrm{B}<$ in $>$ ili-ø ng mangingisda ang bangka 
buy $<$ PFV $>$-UV GEN fisherman NOM boat

«The fisherman bought the boat. » (Schachter \& Otanes, 1972: 70)

Des extraits de textes de littérature orale enregistrés et transcrits auprès des locuteurs de la langue amis ont permis de détailler d'autres aspects de sa morphologie complexe et de sa syntaxe. 